\title{
Potent Therapy and Transcriptional Profile of Combined Erythropoietin-Derived Peptide Cyclic Helix B Surface Peptide and Caspase-3 siRNA against Kidney Ischemia/Reperfusion Injury in Mice
}

\author{
๑ Yuanyuan Wu, ${ }^{1}$ Weiwei Chen, ${ }^{1}$ Yufang Zhang, ${ }^{1}$ Aifen Liu, Cheng Yang, Hui Wang, \\ Tongyu Zhu, Yaping Fan, and BDin Yang \\ Renal Group, Basic Medical Research Centre, Nantong University, Nantong, China (Y.W., Y.Z., A.L.); Leicester-Nantong Joint \\ Institute of Kidney Science, Department of Nephrology, Affiliated Hospital of Nantong University, Nantong, China (W.C., H.W., \\ Y.F., B.Y.); Department of Urology, Zhongshan Hospital, Fudan University, Shanghai, China (C.Y., T.Z.); Shanghai Key Laboratory \\ of Organ Transplantation, Shanghai, China (C.Y., T.Z.); and Renal Group, Department of Cardiovascular Sciences, University of \\ Leicester, University Hospitals of Leicester, Leicester, United Kingdom (Y.W., B.Y.)
}

Received May 8, 2020; accepted July 27, 2020

\begin{abstract}
Cause-specific treatment and timely diagnosis are still not available for acute kidney injury (AKI) apart from supportive therapy and serum creatinine measurement. A novel erythropoietin-derived cyclic helix B surface peptide (CHBP) protects kidneys against AKI with different causes, but the underlying mechanism is not fully defined. Herein, we investigated the transcriptional profile of renoprotection induced by $\mathrm{CHBP}$ and its potential synergistic effects with siRNA targeting caspase-3, an executing enzyme of apoptosis and inflammation (CASP3siRNA), on ischemia/reperfusion (IR)-induced AKI. Utilizing a mouse model with 30-minute renal bilateral ischemia and 48-hour reperfusion, the renoprotection of CHBP or CASP3siRNA was demonstrated in renal function and structure, active caspase-3 and HMGB1 expression. Combined treatment of CHBP and CASP3siRNA further preserved kidney structure and reduced active caspase-3 and HMGB1. Furthermore, differentially expressed genes (DEGs) were identified with fold change $>1.414$ and $P<0.05$. In IR kidneys, 281 DEGs induced by CHBP were mainly involved in promoting cell division and improving cellular function and metabolism (upregulated signal transducer and activator of transcription $5 \mathrm{~B}$ and solute carrier family 22 member 7). The additional administration of CASP3siRNA caused 504 and 418 DEGs in IR + CHBP kidneys with or without negative control small-interfering RNA, with 37
\end{abstract}

genes in common. These DEGs were associated with modulated apoptosis and inflammation (upregulated BCL6, SLPI, and SERPINA3M) as well as immunity, injury, and microvascular homeostasis (upregulated complement factor $\mathrm{H}$ and GREM1 and downregulated ANGPTL2). This proof-of-effect study indicated the potent renoprotection of CASP3siRNA upon CHBP at the early stage of IR-induced AKI. Underlying genes, BCL6, SLPI, SERPINA3M, GREM1, and ANGPTL2, might be potential new biomarkers for clinical applications.

\section{SIGNIFICANCE STATEMENT}

It is imperative to explore new strategies of cause-specific treatment and timely diagnosis for acute kidney injury (AKI). CHBP and CASP3siRNA synergistically protected kidney structure after 48-hour ischemia/reperfusion-induced AKI with reduced injury mediators CASP3 and high mobility group box 1 . CHBP upregulated cell division-, function-, and metabolismrelated genes, whereas CASP3siRNA further regulated immune response- and tissue homeostasis-associated genes. Combined CHBP and CASP3siRNA might be a potent and specific treatment for $\mathrm{AKI}$, and certain dysregulated genes secretory leukocyte peptidase inhibitor and SERPINA3M could facilitate timely diagnosis.

\section{Introduction}

Acute kidney injury (AKI) is a public health problem and has attracted much attention in recent years (Mehta et al., 2016). Worldwide, AKI affects about $2 \%$ of patients in hospital admissions, with a rate of mortality of about $12 \%$, both of

${ }^{1}$ Y.W., W.C., and Y.Z. contributed equally to this work.

This study was supported by the National Natural Science Foundation of China [Grants 81170689, 81570677, and 81873622].

Primary laboratory of origin: Renal Group, Basic Medical Research Centre, Nantong University, Nantong, China.

An earlier version of this paper appears in Research Square under the DOI: 10.21203/rs.3.rs-26803/v1.

https://doi.org/10.1124/jpet.120.000092. which were increased to around $20 \%$ in the intensive care unit (Bouchard et al., 2015; Yang et al., 2015d). There is no specific treatment for AKI apart from passive support or renal replacement therapy, such as volume control or dialysis in clinic (Moore et al., 2018). It is urgent, therefore, to develop specific and effective treatment for AKI to reduce mortality and prevent its progression to chronic kidney disease (Mehta et al., 2015; Noble et al., 2020).

Renal ischemia/reperfusion (IR) injury is a major cause of AKI, characterized by apoptosis, inflammation, and immune response-associated damage (Bellomo et al., 2012; Dong et al., 2019). Recently, the innate repair mechanism in AKI has attracted great attention, which is highlighted by an innate 
repair receptor, a heterodimer of erythropoietin (EPO) receptor and $\beta$ common receptor (EPOR/ $\beta \mathrm{cR}$ ) (Brines and Cerami 2012). $\mathrm{EPO}$, a natural ligand of $\mathrm{EPOR} / \beta \mathrm{cR}$, is defective in tissue protection because of low affinity but has high affinity to a homodimer receptor (EPOR) $)_{2}$ in erythropoiesis (Gobe et al., 2014; Wang et al., 2017; Shi et al., 2018). EPO-derived helix B surface peptide (HBSP) and cyclic HBSP [CHBP, more stable and potent than HBSP (Yang et al., 2014a)] only bind to EPOR/ $\beta c R$, and remain the tissue protective property but without erythropoiesis, so have promising potential for clinical application (Brines et al., 2008; Patel et al., 2012; Wu et al., 2013; Yang et al., 2013). In the IR kidney, CHBP reduces endoplasmic reticulum stress (Zhang et al., 2020) and increases autophagy (Yang et al., 2014a), leading to less apoptosis (Kaushal and Shah 2016). CHBP also ameliorated renal inflammation and reduced chronic deposition of extracellular matrix through inactivating forkhead box O 3 a after IR (Yang et al., 2015a). Nevertheless, the exact underling mechanism in the renoprotection of CHBP is incompletely understood.

Caspase-3, upregulated by IR in the kidney, is a major effector enzyme in the process of apoptosis as well as inflammation (Yang et al., 2011a; Li et al., 2019). Evidence suggests that downregulating the expression of active caspase-3 is presented by $\mathrm{HBSP} / \mathrm{CHBP}$ treatment in IR kidneys (Yang et al., 2013, 2015b). The contributing role of caspase-3 in IR kidneys was further verified by smallinterfering RNA (siRNA), showing that serum-stabilized siRNA targeting caspase-3 greatly reversed renal function and inflammation in a 2-week porcine kidney autotransplantation model (Yang et al., 2014b). It is also intriguing to discover whether there are synergistic effects on IR-induced AKI by combined administration of HBSP/CHBP and caspase-3 siRNA (CASP3siRNA).

In the present study, the effect of CHBP was explored by a single peritoneal injection as well as its cotreatment with CASP3siRNA injected via the tail vein in a 48-hour mouse renal IR model. To delineate the possible mechanisms of single/simultaneous administration, the modern technology of transcriptomic microarray analysis was also used to disclose a transcriptional overview in an array of genes and their biologic involvements.

\section{Materials and Methods}

CHBP. The sequence of CHBP was the same with HBSP, QEQLERALNSS, and it was thioether-cyclized (molecule weight 1416.7). The detailed structure of CHBP was described previously, which was designed and synthesized by Shanghai Institute of Materia Medica, Chinese Academy of Sciences, China (Yang et al., 2014a).

Caspase-3 siRNA. CASP3siRNA, targeting murine caspase-3 mRNA (NCBI CoreNucleotide Accession No. BC038825), were synthesized (Life Technologies, Paisley, UK). The sequences of the CASP3siRNA (Ambion In Vivo, catalog number: 4457309) were sense 5 '-CCUGGUUACUAUUCCUGGAtt- 3 ' and antisense 5 '-UCCAGGAA UAGUAACCAGGtg- ${ }^{\prime}$. The negative control siRNA (NCsiRNA) was also provided by Life Technologies (Ambion In Vivo, catalog number: 4457289), with sequences of sense 5'-UAACGACGCGACGACGUA Att-3' and antisense 5'-UUACGUCGUCGCGUCGUUAtt-3'. Both
CASP3siRNA and NCsiRNA were chemically modified by locked nuclei acid.

Renal IR Surgery. Male C57BL/6 mice, 8-12 weeks, were purchased from the Experimental Animal Center of Yangzhou University, China. All animal experiments were performed according to the guidelines of the Laboratory Animal Monitoring Committee of Jiangsu Province.

The renal IR surgical procedures were performed under general anesthesia by intraperitoneal injection of pentobarbital sodium at $75 \mathrm{mg} / \mathrm{kg}$ body weight (BW). Bilateral kidneys were exposed via dorsal incisions sequentially, and the renal pedicle was carefully isolated and occluded using a nontraumatic vascular clamp for 30 minutes. The efficacy of occlusion was confirmed by the color change of kidney surface, eventually, to dark red. Followed by removing the clamps, patched blanching appeared to the kidney surface and then normal pink, indicating blood reperfusion. Sham operation was performed in a similar manner, except with clamping of renal pedicles. Mice were randomly divided into seven groups ( $n=6)$ as follows: 1 ) sham, 2) IR, 3) IR + CASP3siRNA, 4) IR + NCsiRNA, 5) IR + CHBP, 6) IR + CHBP + CASP3siRNA, and 7) IR + CHBP + NCsiRNA. Having six animals in each group was determined using power calculation according to the change of the key parameter in our previous IR time course model (Zhang et al., 2020) and CHBP intervention study (Yang et al., 2014a). The experimental design was shown in Fig. 1A. A total of $0.03 \mathrm{mg} / \mathrm{kg}$ BW of siRNA (dissolved in saline) was injected into the tail vein 2 hours before surgery. Twenty-four nanomoles per kilogram BW of CHBP (dissolved in saline) was given through intraperitoneal injection at 15 minutes after clamps were released.

Sample Collection. At 48 hours of renal IR injury, animals were anesthetized with pentobarbital sodium, followed by cardiac puncture for drawing whole blood. The serum sample from each animal was then obtained by centrifuging at 10,000 rpm for 15 minutes and stored individually at $-80^{\circ} \mathrm{C}$. Kidneys were removed and transversally cut at the midplane, following crosscutting from the middle. One quarter of each kidney was fixed in $10 \%$ neutral formalin for 24 hours, whereas two quarters were rapidly frozen in liquid nitrogen, and the fourth part was preserved in RNAlater (Life Technologies).

Biochemistry Analysis. The serum creatinine (SCr) level of each animal was determined using a QuantiChrom Creatinine Assay Kit separately (BioAssay Systems, Hayward). Briefly, $30 \mu \mathrm{l}$ of standard or sample serum was transferred into a 96 -well plate followed by adding in $200 \mu \mathrm{l}$ working reagent per well, a mixture of reagent A and B. Absorbance at $510 \mathrm{~nm}$ was read immediately and 5 minutes later. Calculation was performed according to the manufacturer's instruction. The detection was performed three times independently.

Histologic Assessment. Hematoxylin \& eosin (H\&E) staining of kidney tissues was performed to observe and evaluate the degree of tubulointerstitial damage (TID) in the cortex using a scoring system by assessing tubular damage (degeneration and detachment from basement membrane), interstitial expansion (edema or inflammatory cell infiltration), and dilation of tubular lumina. Histologic changes were graded based on the percentage of damaged area involved: $<5 \%$ area was scored $0,5 \%-25 \%$ area was scored $1,25 \%-50 \%$ area was scored $2,50 \%-75 \%$ area was scored 3 , and area exceeding $75 \%$ was scored 4 . Kidney sections were blindly reviewed by two researchers independently. The scores from three compartments (tubular and interstitial areas, tubular lumina) of each kidney were obtained from 12 fields at 200 magnifications. The average scores per field of three compartments were then summed up for each kidney. The final score

ABBREVIATIONS: AEC, 3-amino-9-ethylcarbazole; AKI, acute kidney injury; Angptl2, Angiopoietin-like 2; Bcl6, B cell leukemia/lymphoma 6; BW, body weight; CASP3siRNA, caspase-3 small-interfering RNA; CFH, complement factor $\mathrm{H}$; CHBP, cyclic helix B surface peptide; DEG, differentially expressed gene; EPO, erythropoietin; EPOR/ $\beta \mathrm{cR}$, erythropoietin receptor and $\beta$ common receptor; FC, fold change; GO, Gene Ontology; GREM1, gremlin 1; HBSP, helix B surface peptide; H\&E, hematoxylin \& eosin; HMGB1, high mobility group box 1; IR, ischemia/reperfusion; ISEL, in situ endlabeling; LSD, least significant difference; NCsiRNA, negative control small-interfering RNA; qPCR, quantitative polymerase chain reaction; SCr, serum creatinine; siRNA, small-interfering RNA; SLC22A7, solute carrier family 22 member 7; SLPI, secretory leukocyte peptidase inhibitor; TEC, tubular epithelial cell; TID, tubulointerstitial damage. 

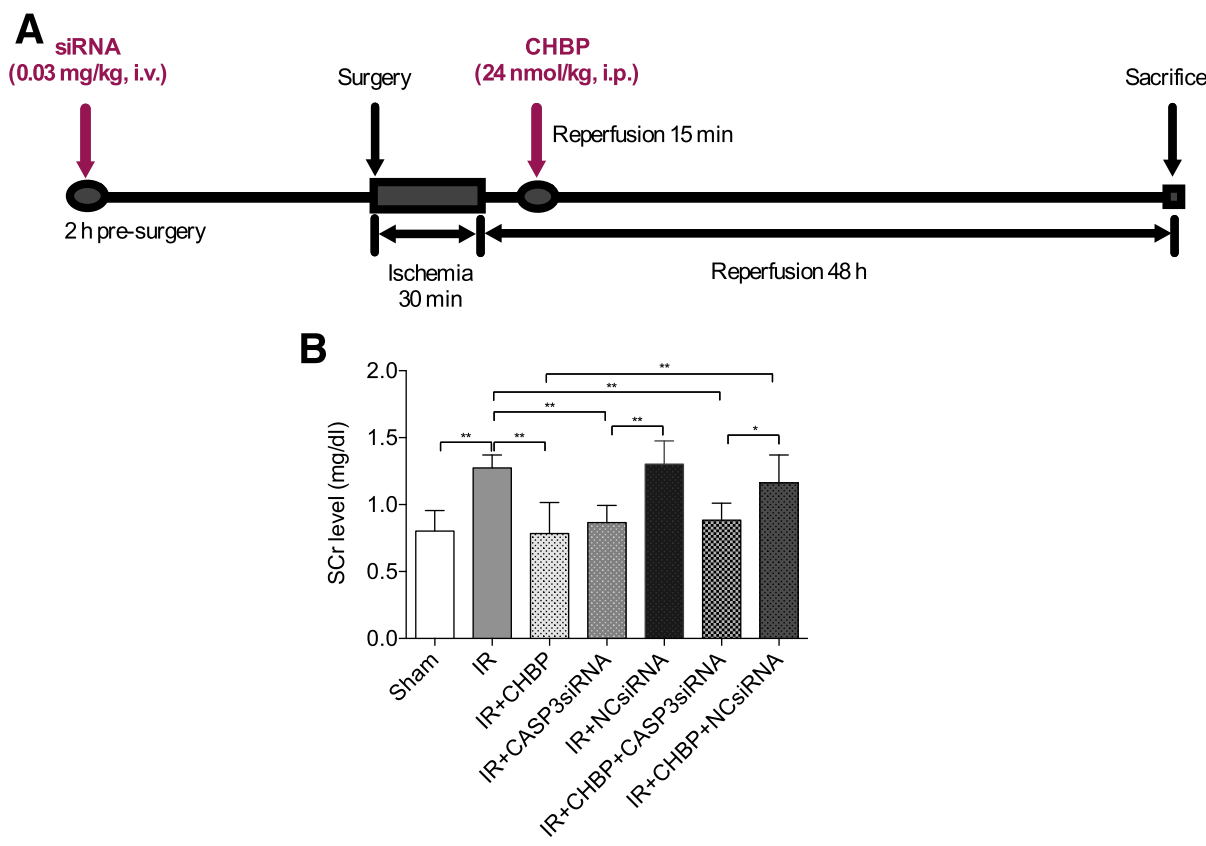

C
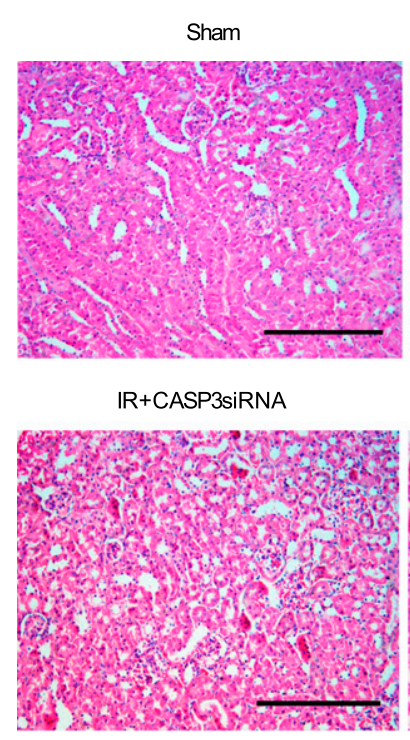

$\mathrm{IR}+\mathrm{CHBP}+\mathrm{NC}$ siRNA

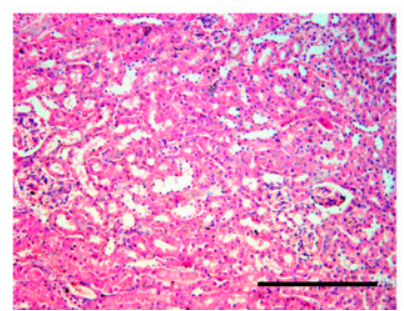

IR+CASP3siRNA
IR

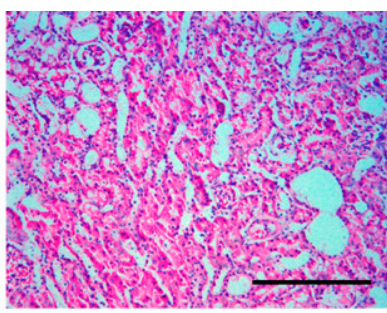

$\mathrm{IR}+\mathrm{NCsiRNA}$

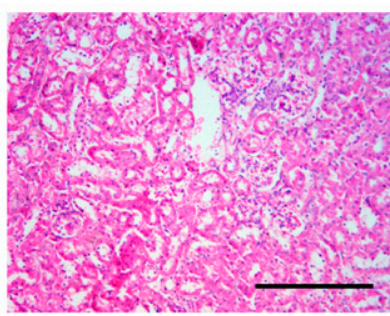

$\mathrm{IR}+\mathrm{CHBP}$

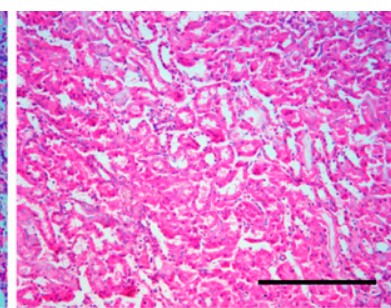

$\mathrm{IR}+\mathrm{CHBP}+\mathrm{CASP} 3 \mathrm{siRNA}$

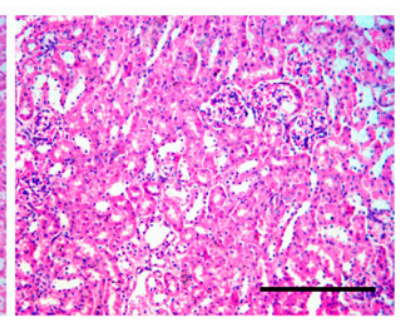

Fig. 1. CHBP and/or CASP3siRNA preserved renal function and structure in IR kidneys. (A) Schematic diagram of mouse renal IR models with the treatment of CHBP and/or CASP3siRNA. Bilateral kidney pedicles were occluded for 30 minutes, followed by 48-hour reperfusion. CASP3siRNA or NCsiRNA was injected via the tail vein at a dose of $0.03 \mathrm{mg} / \mathrm{kg}$ BW 2 hours before surgery. CHBP was given through intraperitoneal cavity at $24 \mathrm{nmol} / \mathrm{kg} \mathrm{BW}$ 15 minutes postreperfusion. (B) The level of $\mathrm{SCr}$ was demonstrated for each group. There were six animals in each group, and the detection was repeated for three times independently. The experiment was performed three times independently. (C) Representative photomicrographs of $\mathrm{H} \& \mathrm{E}$ staining in renal cortex were shown for each group. Scale bar, $100 \mu \mathrm{m}$. (D) Semiquantitative analysis of TID score $(n=6)$. The sections were blindly scored by two researchers independently. Data were shown as means \pm S.D. $n=6$ animals per group; $* P<0.05$; $* * P<0.01$. Statistical comparisons were calculated by ANOVA followed by post hoc LSD test.

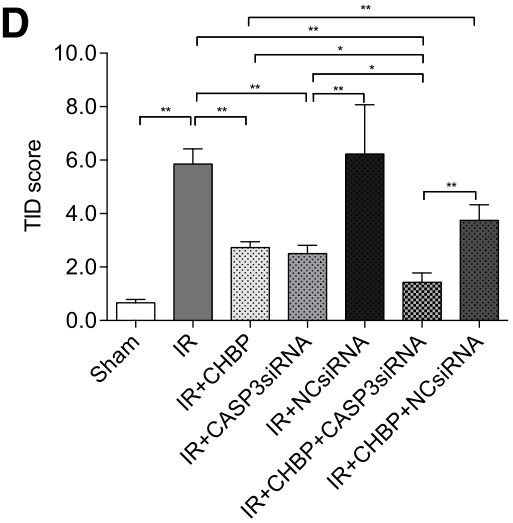


for the animal was then calculated by averaging the scores from left and right kidneys.

In Situ End-Labeling of Apoptotic Cells. Apoptotic cells were detected using a TUNEL Apoptosis Detection Kit (Millipore, MA) by in situ end-labeling (ISEL), as previously described (Wu et al., 2013). Paraffin-embedded kidney sections were dewaxed and digested by proteinase $\mathrm{K}$ at $20 \mu \mathrm{g} / \mathrm{ml}$ for 10 minutes at $37^{\circ} \mathrm{C}$. The sections were then applied with equilibration buffer, terminal deoxynucleotidyl transferase, and anti-digoxigenin-peroxidase sequentially. The labeling of apoptotic cells was then revealed with 3-amino-9-ethylcarbazole (AEC, dark red color). Apoptotic cells were examined at 400 magnifications in up to 20 fields of tubulointerstitial areas in the cortex. The number of positively stained cells in each animal was calculated by averaging the average number per field from left and right kidneys. This was blindly reviewed by two researchers independently.

Immunostaining of Active Caspase-3 in Kidneys. Active $17 \mathrm{kDa}$ subunit of caspase-3 was stained on kidney paraffin sections using the method described before (Yang et al., 2011b). Briefly, sections were dewaxed, and antigen retrieval was performed before incubation with a rabbit-anti-mouse $17 \mathrm{kDa}$ caspase-3 antibody (1:100 dilution; R\&D System, Abingdon, UK). For negative control, normal rabbit immunoglobulin $\mathrm{G}$ was applied at the same concentration of primary antibody. Seventeen kilodalton caspase-3+ cells were counted at 400 magnifications in up to 20 cortical fields of each kidney blindly by two researchers independently. The number of apoptotic cells for each animal was obtained by averaging the numbers from all fields in both kidneys.

Western Blot Analysis. Twenty-five micrograms of kidney homogenate was separated in reduced SDS-PAGE gels and electroblotted onto a polyvinylidene fluorid membrane. The membrane was then blocked in $5 \%$ (weight/volume) nonfat milk, followed by probing with an anti-full length caspase-3 antibody (CST, Danvers, MA) at 1: 400 dilution, an anti-high mobility group box 1 (HMGB1) antibody (CST) at 1:1000, or an anti- $\beta$-actin antibody (Abcam, Cambridge, UK) at 1:8000 dilution for overnight at $4^{\circ} \mathrm{C}$. The corresponding secondary antibody (Jackson ImmunoResearch Laboratories, West Grove) was then applied to the membrane for 2 hours at room temperature. Afterward, antibody binding was revealed using enhanced chemiluminescence substrate (Thermo Scientific, Waltham, MA) and a Molecular Imager Chemi Doc XRS + system (Bio-Rad, Berkeley). The experiment was performed three times independently.

Microarray Analysis. For the kidney stored in RNAlater, microarray analysis was performed to reveal the profile of whole genomic transcripts by Shanghai Biotechnology Corporation, China. The detection was done in four groups $(n=3)$ : IR, IR + CHBP, IR + CHBP + CASP3siRNA, and IR + CHBP + NCsiRNA. The chosen kidney tissue samples from three animals in each group were nearest to the average level of the group in renal function and structure. The total 12 kidney samples were analyzed individually. The RNA integrity and quantity were monitored by the 2100 bioanalyzer (Agilent Technologies, Santa Clara, CA) and NanoDrop One (Thermo Scientific), respectively. Two micrograms RNA with an Integrity Number of no less than eight was required for the genomic profile analysis. The Agilent Whole Mouse Genome Oligo Microarray was applied to interrogate about 41,174 transcripts targeting 34,000 well established annotated genes. The criteria of fold change (FC) $>1.414$ (upregulated genes) or $\mathrm{FC}<-1.414$ (downregulated genes) and $P<0.05$ was used for sorting significant differentially expressed genes (DEGs). The cutoff value of FC was based on the fact that 0.5 cycle was the minimum number of polymerase chain reaction (PCR) cycle to distinguish the expressional differences between two samples.

Validation of Candidate DEGs by Quantitative PCR. Total RNAs were extracted by Trizol reagent from the kidney tissues of the same animals selected for microarray analysis. One microgram total RNA was used for reverse transcription in a $20-\mu$ l reaction system supplemented with $4 \mu \mathrm{l}$ of 5x HiScript II qRT SuperMix and RNasefree water using a kit of HiScript II Q RT SuperMix for quantitative PCR (qPCR) (Vazyme, Nanjing, China). The temperature setting was $50^{\circ} \mathrm{C}$ for 15 minutes, followed by $85^{\circ} \mathrm{C}$ for 2 minutes. One microliter of cDNA product was amplified within an SYBR reaction system (Bioline, London, UK) containing $200 \mathrm{nM}$ forward and reverse primers (Table 1, Biomics, Nantong, China) at $95^{\circ} \mathrm{C}$ for 10 minutes followed by 40 cycles of $95^{\circ} \mathrm{C}$ for 15 seconds and $55^{\circ} \mathrm{C}$ for 60 seconds. The level of $\beta$-actin mRNA was used as an endogenous control.

Gene Function Analysis. Functional enrichment analysis of significant DEGs identified between groups was performed using Gene Ontology (GO; http://geneontology.org/) (Harris et al., 2004). The resulting GO terms with $P$ values less than 0.05 were considered significantly enriched.

Statistical Analysis. Data were expressed as mean \pm S.D. The statistical analyses of the data were performed using IBM SPSS Statistics version 26.0 software. One-way ANOVA analysis was used to check the homogeneity of variance, and then post hoc least significant difference (LSD) test was used for multiple comparisons. The data of qPCR were analyzed using a two-tailed unpaired Student's $t$ test between two groups. Statistical significance was defined as $P<0.05$.

\section{Results}

Improved Kidney Function and Structure. At 48 hours, the $\mathrm{SCr}$ level raised by IR was significantly reduced by the treatment of CHBP $(1.27 \pm 0.09$ vs. $0.78 \pm 0.23, P<0.01)$, CASP3siRNA $(1.27 \pm 0.09$ vs. $0.86 \pm 0.12, P<0.01)$, or CHBP + CASP3siRNA $(1.27 \pm 0.09$ vs. $0.88 \pm 0.12, P<0.01$, Fig. 1B). However, no significant difference was observed among these treatments. IR mice treated with CASP3siRNA or CHBP + CASP3siRNA demonstrated a significantly lower SCr level than those treated with NCsiRNA $(0.86 \pm 0.12 \mathrm{vs}$. $1.30 \pm 0.71, P<0.01)$ or CHBP + NCsiRNA $(0.88 \pm 0.12$ vs. $1.16 \pm 0.20, P<0.05)$, respectively.

Representative images for H\&E staining were chosen to show the damage in different parts of the cortex, such as the tubular area, the tubular lumen, and the interstitial area (Fig. 1C). CHBP, CASP3siRNA, and CHBP + CASP3siRNA treatment significantly decreased the extent of TID in IR kidneys $(2.72 \pm 0.21$ or $2.49 \pm 0.30$ or $1.43 \pm 0.34$ vs. $5.85 \pm$ 0.56 , all $P<0.01$, Fig. 1D). Furthermore, IR mice with cotreatment of CHBP and CASP3siRNA exhibited a lower level of TID in contrast to those treated with CHBP only or CASP3siRNA only ( $1.43 \pm 0.34$ vs. $2.72 \pm 0.21$ or $2.49 \pm 0.30$, both $P<0.05)$. In addition, CASP3siRNA-treated IR or IR + CHBP mice demonstrated a significant decrease of TID compared with corresponding NCsiRNA controls $(2.49 \pm 0.30$ vs. $6.22 \pm 1.83 ; 1.43 \pm 0.34$ vs. $3.74 \pm 0.58$, both $P<0.01$ ).

Alleviated Apoptosis. As antiapoptosis is a shared renoprotective effect used by CHBP and CASP3siRNA, immunolabeling was performed to examine their effectiveness on reducing kidney IR-induced apoptosis. Representative images of apoptotic cells were chosen to present the morphology and localization of apoptotic cells (Fig. 2A). IR significantly raised the number of ISEL+ cells compared with sham controls $(0.72 \pm 0.05$ vs. $0.05 \pm 0.01, P<0.01)$ but greatly lowered by CHBP, CASP3siRNA, and CHBP + CASP3siRNA $(0.72 \pm$ 0.05 vs. $0.25 \pm 0.06$ or $0.22 \pm 0.06$ or $0.21 \pm 0.05$, all $P<0.01$, Fig. 2B). Nevertheless, comparable levels of ISEL+ cells were found among these treatments. In contrast to NCsiRNA controls, CASP3siRNA reduced apoptosis in either IR kidneys $(0.77 \pm 0.22$ vs. $0.22 \pm 0.06, P<0.01)$ or CHBP-modified IR kidneys $(0.41 \pm 0.09$ vs. $0.21 \pm 0.05, P<0.01)$.

Decreased Active Caspase-3 Staining Positive Cells. Cells labeled with active $17 \mathrm{kDa}$ caspase-3 often having the 
TABLE 1

The sequence of primers for $\mathrm{qPCR}$

\begin{tabular}{|c|c|c|}
\hline Gene Symbol & Full Name & Primers $\left(5^{\prime}-3^{\prime}\right)$ \\
\hline SLC22A7 & Solute carrier family 22 member 7 & $\begin{array}{l}\text { Forward: CTGTCTGCCTGTGTTTATCC } \\
\text { Reverse: CTTCCCCAAATGCCACAGCT }\end{array}$ \\
\hline $\mathrm{CFH}$ & Complement factor $\mathrm{H}$ & $\begin{array}{l}\text { Forward: ACTTTCTCAGATTTTCCTGG } \\
\text { Reverse: TGGTTGTTACATGCTTTGGG }\end{array}$ \\
\hline ANGPTL2 & Angiopoietin-like 2 & $\begin{array}{l}\text { Forward: GGATGGTTCACAGAGAGAGTAC } \\
\text { Reverse: CTCCTTGGAGTTGACACAAATG }\end{array}$ \\
\hline GREM1 & Gremlin-1 & $\begin{array}{l}\text { Forward: GCAAGTATCTGAAGVGAGATTG } \\
\text { Reverse: CGTCATGGTGGTGAACTTCTTG }\end{array}$ \\
\hline ACTB & $\beta$-actin & $\begin{array}{l}\text { Forward: GAGACCTTCAACACCCCAGC } \\
\text { Reverse: ATGTCACGCACGATTTCCC }\end{array}$ \\
\hline
\end{tabular}

morphologic features of apoptosis as condensed nuclei were mainly located in tubular epithelia, tubular lumina, and interstitial areas (Fig. 3A). The number of active caspase-3+ cells was significantly increased by IR $(0.66 \pm 0.15$ vs. $0.01 \pm$ $0.02, P<0.01)$ but decreased by CHBP, CASP3siRNA, and CHBP + CASP3siRNA $(0.66 \pm 0.15$ vs. $0.13 \pm 0.03$ or $0.32 \pm$ 0.15 or $0.17 \pm 0.06, P<0.05$ or 0.01 , Fig. $3 \mathrm{~B})$. No significant differences between these treatments were observed. Comparing with NCsiRNA, CASP3siRNA significantly reduced the number of $17 \mathrm{kDa}$ caspase-3+ cells in IR kidneys (1.07 \pm 0.51 vs. $0.32 \pm 0.15, P<0.01$ ).
Decreased Expression of Active Caspase-3 and HMGB1 Protein. Western blotting was used to determine whether there is a coeffect of CHBP and CASP3siRNA on the expression of $17 \mathrm{kDa}$ active caspase- 3 . The level of $17 \mathrm{kDa}$ caspase3 was significantly increased by $\operatorname{IR}(0.90 \pm 0.34$ vs. $0.57 \pm 0.23, P<$ 0.05) but reduced by CHBP, CASP3siRNA, and CHBP + CASP3siRNA $(0.90 \pm 0.34$ vs. $0.40 \pm 0.12$ or $0.52 \pm 0.19$ or $0.29 \pm 0.08$, $P<0.01$ or 0.05 , Fig. $4, \mathrm{~A}$ and B). IR mice with the cotreatment of CHBP and CASP3siRNA showed an even lower expression of $17 \mathrm{kDa}$ caspase-3 compared with IR mice treated with CASP3siRNA $(0.29 \pm 0.08$ vs. $0.52 \pm 0.19, P<0.05)$. Controlled by
A

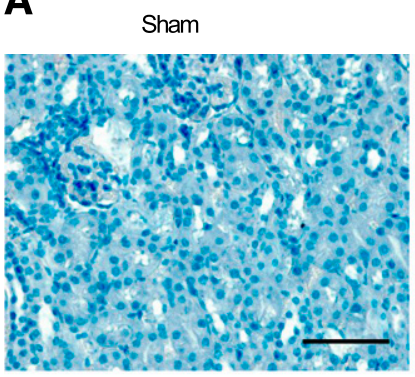

IR+CASP3siRNA

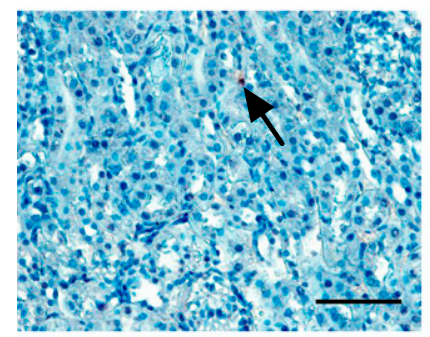

$\mathrm{IR}+\mathrm{CHBP}+\mathrm{NC}$ SiRNA

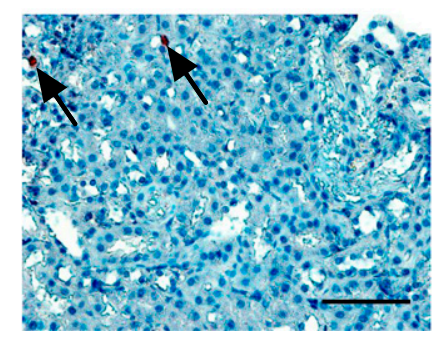

IR

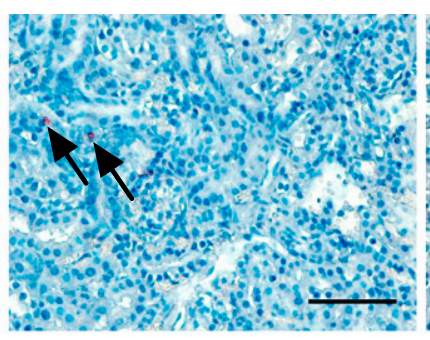

IR+NCsiRNA

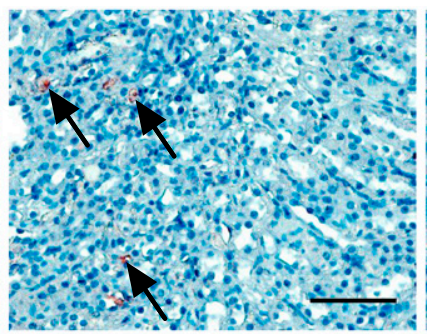

B

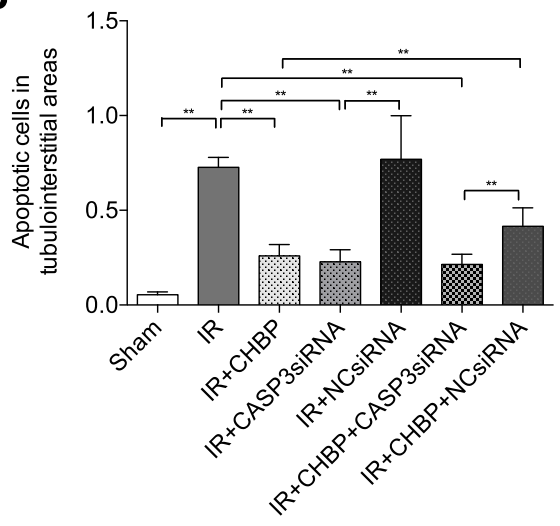

Fig. 2. CHBP and/or CASP3siRNA ameliorated apoptosis in tubulointerstitial areas. (A) The method of ISEL fragmented DNAs was used to detect apoptotic cells in kidney tissues. AEC was used to develop color labeling. Representative photomicrographs of apoptotic cells (indicated by arrows) in cortical areas were shown in each group. Scale bar, $50 \mu \mathrm{m}$. (B) The average number of ISEL+ cells per field are demonstrated for each group $(n=6)$. The sections were blindly reviewed by two researchers independently. Data were shown as means \pm S.D. $n=6$ animals per group; $* P<0.05$; $* * P<0.01$. Statistical comparisons were calculated by ANOVA followed by post hoc LSD test. 
A

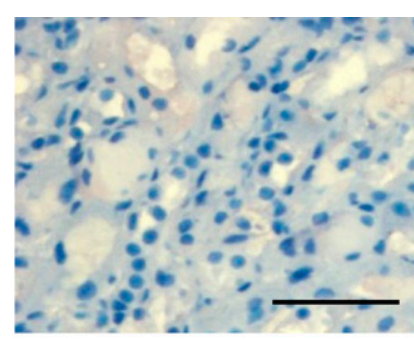

IR+CASP3siRNA

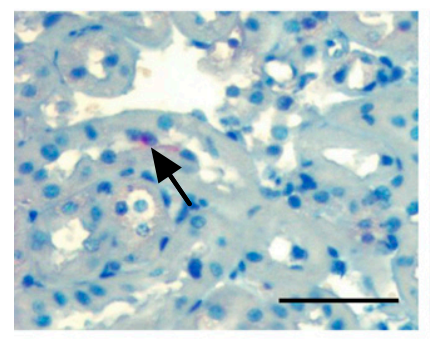

$\mathrm{IR}+\mathrm{CHBP}+\mathrm{NCsiRNA}$

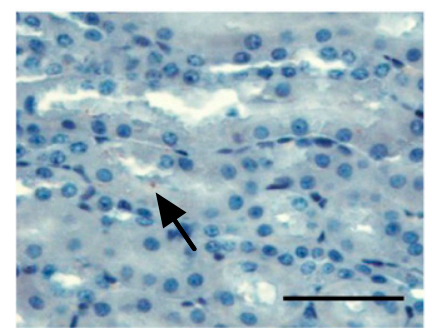

IR

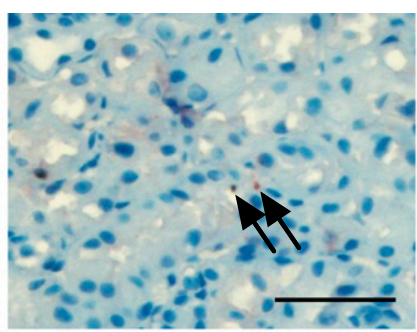

IR+NCsiRNA

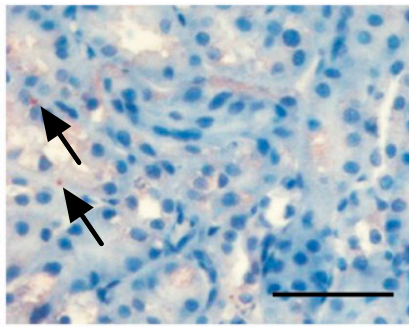

$\mathrm{IR}+\mathrm{CHBP}$

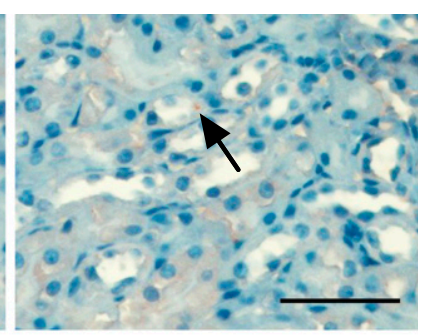

$\mathrm{IR}+\mathrm{CHBP}+\mathrm{CASP} 3 \mathrm{siRNA}$

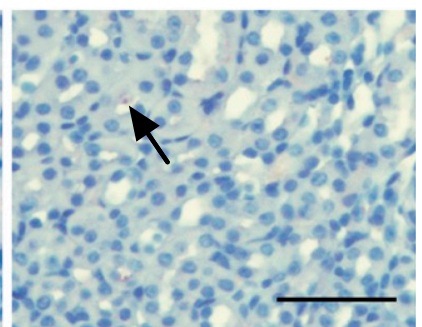

B

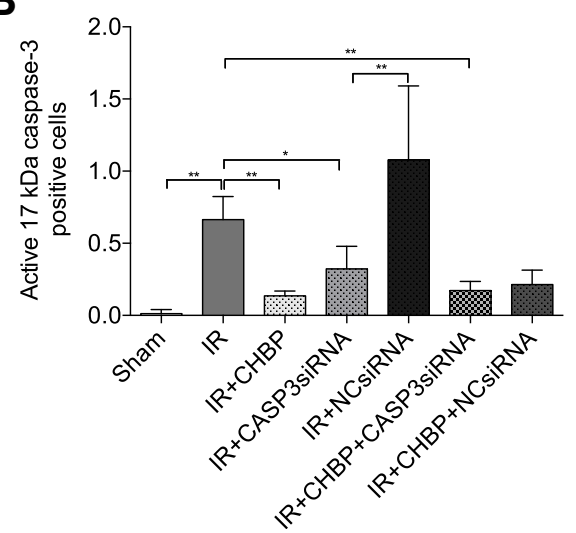

Fig. 3. CHBP and/or CASP3siRNA decreased the number of active $17 \mathrm{kDa}$ caspase-3-positive cells. (A) Representative photomicrographs of cells positively stained with $17 \mathrm{kDa}$ caspase-3+ were shown in the indicated groups. Detected by immunostaining, $17 \mathrm{kDa}$ caspase-3 was labeled and revealed by AEC. Scale bar, $50 \mu \mathrm{m}$. (B) The average number of $17 \mathrm{kDa}$ caspase-3+ cells per field are demonstrated in each group $(n=6)$. The sections were blindly reviewed by two researchers independently. Data were shown as means \pm S.D. $n=6$ animals per group; $* P<0.05 ; * * P<0.01$. Statistical comparisons were calculated by ANOVA followed by post hoc LSD test.
NCsiRNA, $17 \mathrm{kDa}$ caspase-3 was reduced by CASP3siRNA in IR + CHBP kidneys $(0.59 \pm 0.23$ vs. $0.29 \pm 0.08, P<0.05)$.

HMGB1 is a proinflammatory factor known to arouse profound innate responses by binding to toll-like receptor 4 on surface of tubular epithelial cells (TECs) and macrophages (Wu et al., 2010; Chen et al., 2017). Western blotting using kidney homogenates demonstrated IR significantly increased HMGB1 expression compared with sham controls ( $3.21 \pm 1.41$ vs. $1.51 \pm$ $0.67, P<0.01$, Fig. 4, C and D). However, treatments with CHBP, CASP3siRNA, and CHBP + CASP3siRNA decreased the high level of HMGB1 $(1.09 \pm 0.63$ or $1.57 \pm 0.59$ or $0.81 \pm$ 0.40 vs. $3.21 \pm 1.41$, all $P<0.01$ ). Moreover, IR mice with cotreatment of CHBP and CASP3siRNA exhibited an even lower level of renal HMGB1 compared with CASP3siRNAtreated animals $(0.81 \pm 0.40$ vs. $1.57 \pm 0.59, P<0.05)$. In contrast to NCsiRNA, CASP3siRNA reduced HMGB-1 expression in IR kidneys $(4.65 \pm 0.86$ vs. $1.57 \pm 0.59, P<0.01)$ as well as in IR + CHBP kidneys $(2.63 \pm 1.26$ vs. $0.81 \pm 0.40, P<0.01)$.

Identification of Differentially Expressed Genes and Revalidation. To disclose the mechanism of renoprotection induced by CHBP and/or CASP3siRNA, transcriptomic microarray analysis was conducted to identify DEGs affected in the IR kidneys. The three chosen samples from each group could best represent biochemistry and pathologic changes in the group.
Two hundred eighty-one DEGs (153 upregulated, 128 downregulated) were identified in the CHBP-treated IR kidneys versus IR kidneys (Fig. 5A). Four hundred eighteen DEGs (226 upregulated, 192 downregulated) were shown by the additional administration of CASP3siRNA to CHBP-treated IR kidneys versus IR + CHBP kidneys, with 46 genes in common with the comparison of IR + CHBP versus IR groups. In contrast to the NCsiRNA treatment to IR + CHBP kidneys, CASP3siRNA produced 504 DEGs (218 upregulated, 286 downregulated) in IR + CHBP kidneys, of which nine genes were commonly altered with the IR + CHBP kidneys versus IR kidneys and 37 genes in common with the comparison of IR + CHBP + CASP3siRNA versus IR + CHBP. Among the above three comparisons, there were only three genes affected universally. The top five genes upregulated and downregulated in three comparisons are listed (Tables 2-4). Among DEGs, upregulated BCL6 was associated with the negative regulation of apoptosis (Table 2), upregulated secretory leukocyte peptidase inhibitor (SLPI) and SERPINA3M were related to inflammation (Table 3), and upregulated GREM1 and downregulated ANGPTL2 were linked to injury, inflammation, and microvascular homeostasis (Table 4).

To validate the outcome of microarray analysis, four DEGs were selected for qPCR detection: upregulated SLC22A7 by CHBP compared with the IR group (FC = 2.996), associated 
A

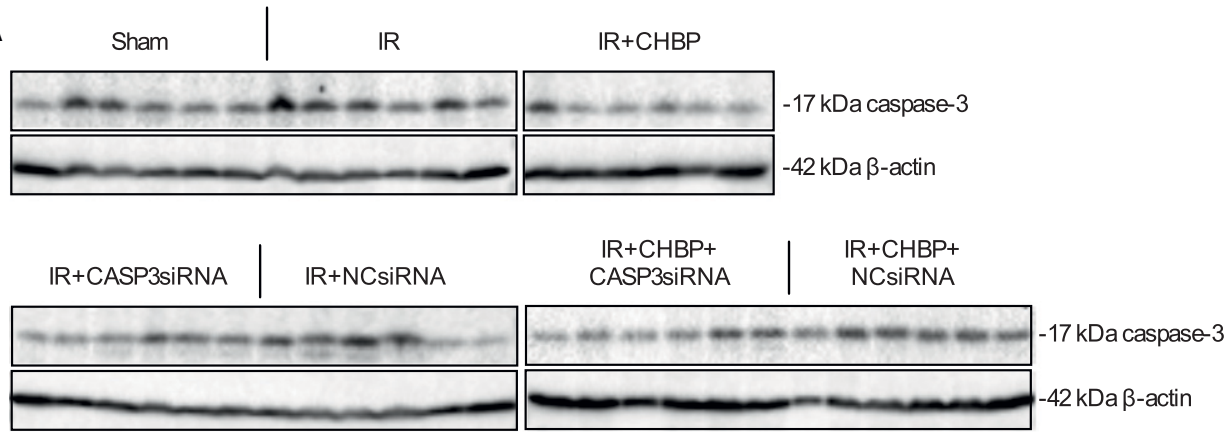

B

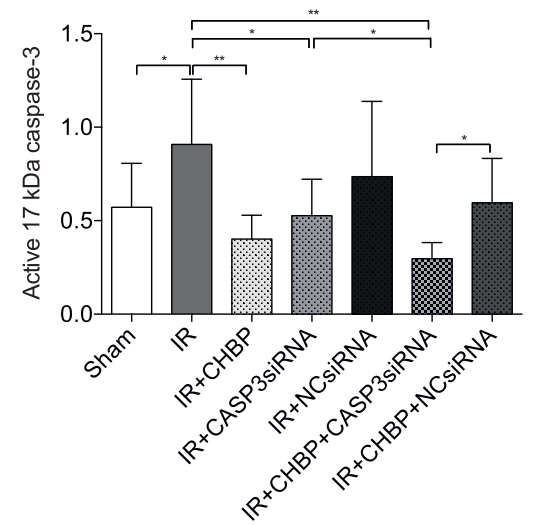

C

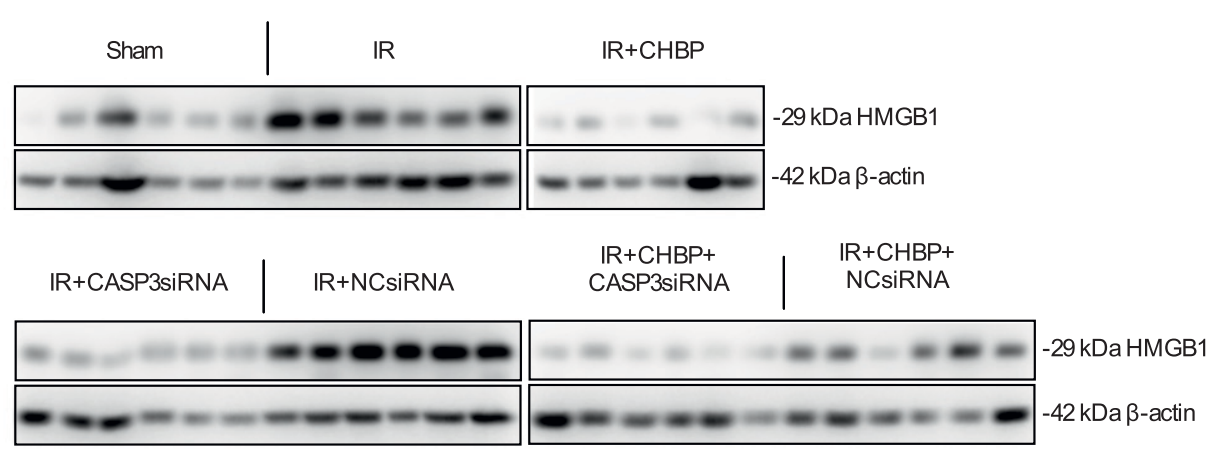

Fig. 4. CHBP and/or CASP3siRNA decreased $17 \mathrm{kDa}$ active caspase-3 and HMGB1 expression in IR kidneys. (A) The level of active caspase-3 was measured by Western blotting and typical bands were shown. (B) Semiquantitative analysis showed the expression of $17 \mathrm{kDa}$ caspase-3 corrected by the endogenous control of $\beta$-actin in each group $(n=6)$. (C) Representative bands of HMGB1 were shown. (D) The level of HMGB1 protein corrected by $\beta$-actin was determined in each group $(n=6)$. The immunoblotting was performed three times independently. Data were shown as means \pm S.D. $n=6$ animals per group; $* P<0.05$; $* * P<0.01$. Statistical comparisons were calculated by ANOVA followed by post hoc LSD test.

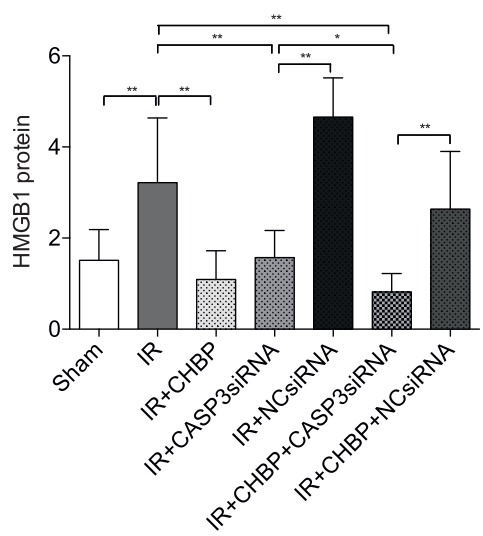

with the epithelial function of organic anion transport; upregulated CFH by CASP3siRNA compared with NCsiRNA $(\mathrm{FC}=1.949)$, a negative regulator in the alternative pathway of complement activation; and ANGPTL2 and GREM1 as described above. qPCR results showed that the level of
SLC22A7 was greatly upregulated by CHBP (Fig. 5B); CFH and GREM1 were increased (Fig. 5, C and D), but Angptl2 was decreased by CASP3siRNA compared with NCsiRNA (Fig. 5E). Thus, all results from qPCR were consistent with the output of microarray data. 
A

IR+CHBP versus IR
(281 significant DEGs)
$\mathrm{IR}+\mathrm{CHBP}+\mathrm{CASP} 3$ siRNA versus $\mathrm{IR}+\mathrm{CHBP}$

(418 significant DEGs)

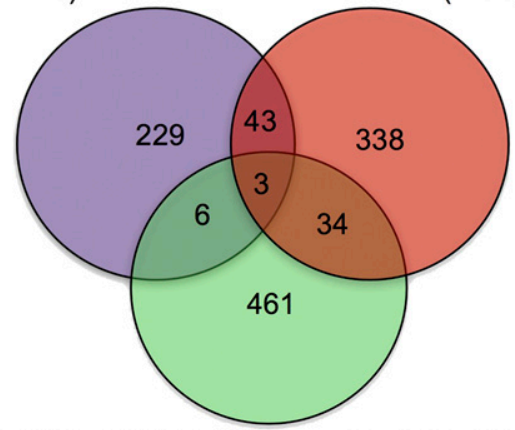

$\mathrm{IR}+\mathrm{CHBP}+\mathrm{CASP} 3$ siRNA versus $\mathrm{IR}+\mathrm{CHBP}+\mathrm{NC}$ SiRNA (504 significant DEGs)
B

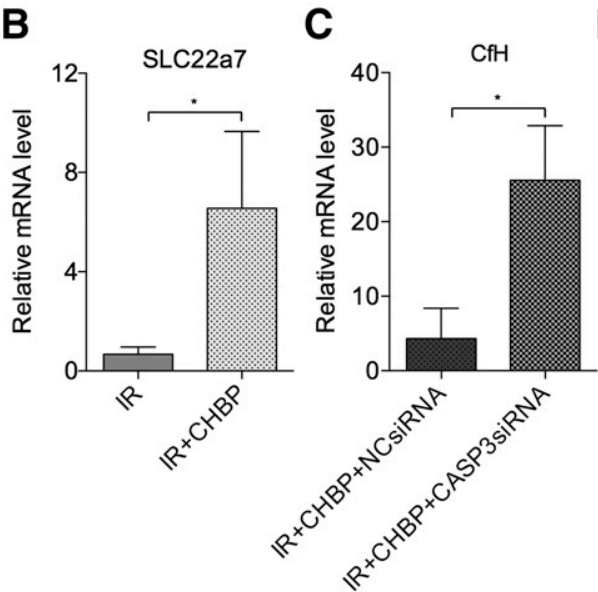

E

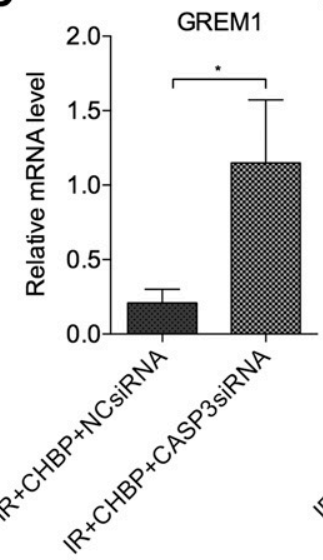

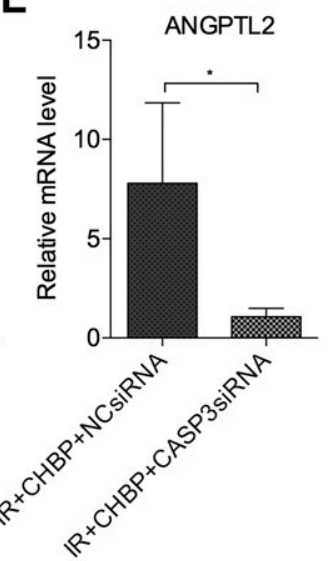

Fig. 5. Identified DEGs and $q P C R$ validation. (A) Venn diagram illustrating the number of significant DEGs in the three comparisons. The sorting criteria was FC $>1.414$ and $P<0.05$. In four groups $(n=3)$, each kidney sample was analyzed separately. (B-E) The expression of SLC22A7, CFH, GREM1, and ANGPTL2 mRNA was detected by qPCR, which was done three times independently. $n=3$ kidney samples from individual animals in each group; $* P<0.05$. Statistical comparisons were calculated by twotailed unpaired Student's $t$ test.
GO Analysis of the DEGs. The identified DEGs were subjected to GO functional enrichment analysis to elucidate biologic processes altered by CHBP and/or CASP3siRNA in the IR kidneys at 48 hours. Top 30 items of biologic process $(P<0.05)$ with enrich factors are presented (Fig. 6). DEGs induced by CHBP were mainly involved in positive regulation of mitotic cell cycle, regulation of protein tyrosine kinase activity, acyl-CoA/glucose/cholesterol metabolic processes, positive regulation of cellular component biogenesis, and organic anion transport (Fig. 6A). For instance, CHBP upregulated signal transducer and activator of transcription $5 \mathrm{~B}(\mathrm{FC}=1.478)$, a positive regulator of mitotic cell cycle, and SLC22A7, mediating organic anion transport, and also a positive regulator of cellular component biogenesis and glucose metabolic process. Further altered genes by CASP3siRNA treatment in IR + CHBP kidneys versus IR + CHBP were involved in the negative regulation of immune response (Fig. 6B). Compared with the NCsiRNA control, CASP3siRNA further affected biologic processes, including regulation of interleukin-1- $\beta$ production, positive regulation of cAMP metabolic process, positive regulation of

TABLE 2

IR + CHBP vs. IR, top five upregulated and downregulated genes

The gene in italic was discussed for its potential function in IR-induced AKI. $P<0.05$.

\begin{tabular}{|c|c|c|c|}
\hline $\begin{array}{l}\text { Accession Number } \\
\text { Upregulated }\end{array}$ & Gene Symbol & Gene Name & Fold Change \\
\hline AK016105 & 4930553I04RIK & RIKEN cDNA 4930553 I04 gene & 8.559 \\
\hline AK046501 & SACS & Sacsin & 5.261 \\
\hline NM_013501 & CRYAA & Crystallin, alpha A & 4.357 \\
\hline NM_011255 & RBP4 & Retinol binding protein 4 , plasma & 4.213 \\
\hline NM_009744 & $B C L 6$ & B cell leukemia /lymphoma 6 & 3.622 \\
\hline \multicolumn{4}{|l|}{ Downregulated } \\
\hline NM_001167777 & ASXL3 & Additional sex combs like 3 & -24.005 \\
\hline NM 029070 & CLDN26 & Claudin 26 & -5.985 \\
\hline NM_001167746 & DNAH17 & Dynein, axonemal, heavy chain 17 & -5.922 \\
\hline AK171340 & PITPNC1 & Phosphatidylinositol transfer protein, cytoplasmic 1 & -5.113 \\
\hline NM_007501 & NEUROD4 & Neurogenic differentiation 4 & -5.094 \\
\hline
\end{tabular}


TABLE 3

IR + CHBP + CASP3siRNA vs. IR + CHBP, top five upregulated and downregulated genes

The genes in italic were discussed for their potential function in IR-induced AKI. $P<0.05$.

\begin{tabular}{|c|c|c|c|}
\hline $\begin{array}{l}\text { Accession Number } \\
\text { Upregulated }\end{array}$ & Gene Symbol & Gene Name & Fold Change \\
\hline NM_001001450 & SSXB2 & Synovial sarcoma, X member B, breakpoint 2 & 14.730 \\
\hline NR_028111 & 4930523C07RIK & RIKEN cDNA 4930523C07 gene & 10.131 \\
\hline NM_011414 & SLPI & Secretory leukocyte peptidase inhibitor & 8.478 \\
\hline NM_009253 & SERPINA3M & Serine (or cysteine) peptidase inhibitor, clade A, member $3 M$ & 8.289 \\
\hline NM_175309 & UPK3B & Uroplakin 3B & 5.483 \\
\hline \multicolumn{4}{|l|}{ Downregulated } \\
\hline AK016105 & 4930553I04RIK & RIKEN cDNA 4930553104 gene & -8.735 \\
\hline NM_029747 & 2410137M14RIK & RIKEN cDNA 2410137M14 gene & -5.134 \\
\hline NM_147025 & OLFR380 & Olfactory receptor 380 & -4.354 \\
\hline NM_134160 & MCOLN3 & Mucolipin 3 & -4.142 \\
\hline NM_001163513 & DLG5 & Discs large MAGUK scaffold protein 5 & -3.700 \\
\hline
\end{tabular}

phosphatidylinositol 3-kinase signaling, and release of cytochrome c from mitochondria (Fig. 6C).

\section{Discussion}

The present study demonstrated that a single dose of CHBP or CASP3siRNA markedly ameliorated IR-induced kidney injury in terms of preserving renal function and structure, reducing active caspase- 3 and HMGB1 expression. The combination of both further decreased TID, active caspase-3, and HMGB1. In addition, genomic microarray analysis identified DEGs induced by CHBP that were mainly involved in preserving cell division, cellular function, and metabolism. DEGs modified by CASP3siRNA were associated with inhibiting inflammation and maintaining vascular function. Certain genes such as BCL6, SLPI, SERPINA3M, GREM1, and ANGPTL2 might be potential biomarkers in IR-induced AKI.

The present study demonstrated that a single dose of CHBP, with a plasma half-life of 300 minutes (Yang et al., 2014a), administered 15 minutes after reperfusion greatly ameliorated renal IR injury at the early stage of 48 hours. This result was consistent with the evidence that a single dose of CHBP protected the kidney from IR injury at 12 weeks (Yang et al., 2015a). Linear HBSP, with a plasma half-life of about 2 minutes, administered at 1,6 , and 12 hours protected the kidney against IR injury at 24 hours (Brines et al., 2008). Our previous study also showed that daily injection of HBSP protected the kidney from immunosuppressant cyclosporine A-induced damage upon IR injury but did not affect IR injury alone in a 2 -week rat model (Wu et al., 2013). It has been also reported that CHBP protected against aristolochic acidinduced AKI (Zeng et al., 2017). These data imply a variety of potential clinical applications of CHBP or HBSP.

It is the first time verifying that a single dose of CASP3siRNA was comparable to CHBP in renal protection. siRNA is a potent and specific tool that can silence detrimental genes under disease conditions, so siRNA therapy provides perspective in the development of precision medicine (Hawgood et al., 2015). Although there are over 30 siRNA-related clinical trials that have been completed, no siRNA treatment against AKI is available in clinical practice. The result from this study implies that caspase-3 gene may be one of the major affected genes by CHBP in renoprotection; therefore, CASP3siRNA might be an alternative treatment in addition to CHBP for IR-induced renal injury.

The transcriptomic profile, moreover, demonstrated that CHBP-altered genes in biologic processes were mainly linked to cell division, cellular function, and metabolism. For example, signal transducer and activator of transcription $5 \mathrm{~B}$ upregulated by CHBP was involved in cell proliferation in rodent kidneys (Chen et al., 2007; Fragiadaki et al., 2017), whereas SLC22A7, enriched in organic anion transport, was associated with the extrusion of creatinine from TECs and maintaining SCr level (Shen et al., 2015). BCL6, among the top five DEGs upregulated by CHBP (Table 2), has a broad role on antiapoptosis and cell survival (Baron et al., 2010), promoting the expression of organic anion transporter 1 in TECs and maintaining the secreting function of TECs (Wegner et al., 2014). In addition, metabolic processes were greatly enriched by CHBP, including glucose metabolism, which is beneficial for energy production (Wei et al., 2014). It has also been

TABLE 4

IR + CHBP + CASP3siRNA vs. IR + CHBP + NCsiRNA, top five upregulated and downregulated genes

Genes in italic indicate analysis by $\mathrm{qPCR}$ and were discussed as biomarker candidates of IR-induced AKI. $P<0.05$

\begin{tabular}{|c|c|c|c|}
\hline $\begin{array}{l}\text { Accession Number } \\
\text { Upregulated }\end{array}$ & Gene Symbol & Gene Name & Fold Change \\
\hline NM_001001450 & SSXB2 & Synovial sarcoma, X member B, breakpoint 2 & 14.817 \\
\hline NM_007729 & COL11A1 & Collagen, type XI, alpha 1 & 8.978 \\
\hline \multicolumn{4}{|l|}{ Downregulated } \\
\hline AK041888 & ANGPTL2 & Angiopoietin-like 2 & -24.390 \\
\hline NM_026648 & DNAAF1 & Dynein, axonemal assembly factor 1 & -6.579 \\
\hline
\end{tabular}


A

$\operatorname{IR}+\mathrm{CHBP}(\mathrm{n}=3)$ versus IR $(\mathrm{n}=3)$

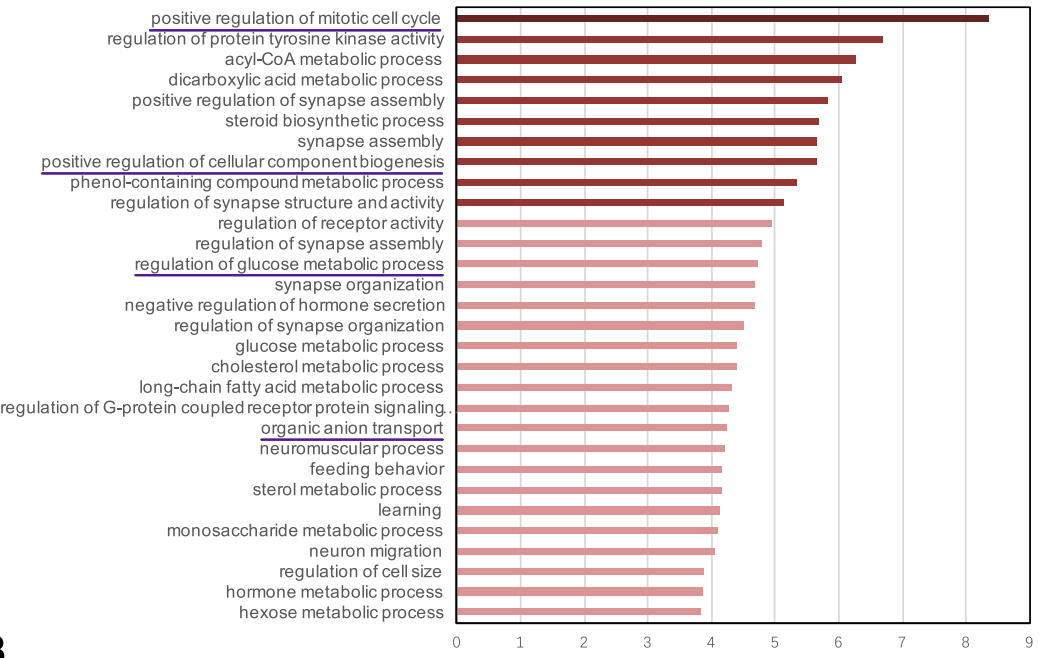

B

\section{$\mathrm{IR}+\mathrm{CHBP}+\mathrm{CASP} 3 \mathrm{siRNA}(\mathrm{n}=3)$ versus $\mathrm{IR}+\mathrm{CHBP}(\mathrm{n}=3)$}

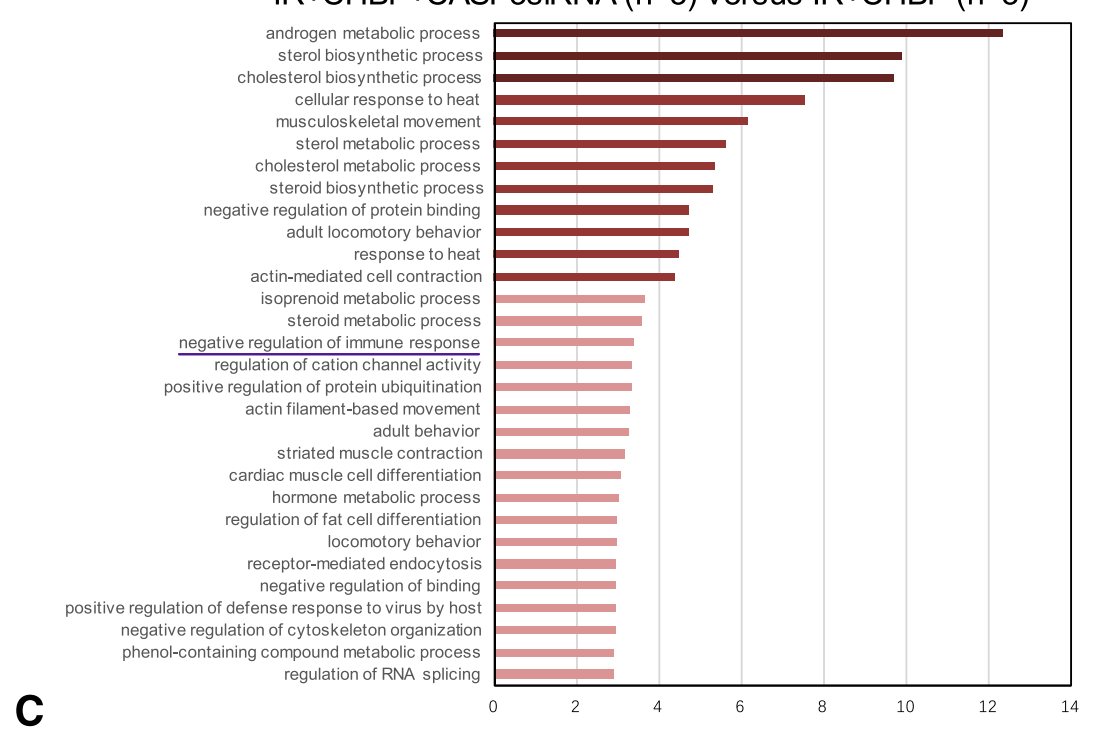

$\mathrm{IR}+\mathrm{CHBP}+\mathrm{CASP} 3 \mathrm{siRNA}(\mathrm{n}=3)$ versus $\mathrm{IR}+\mathrm{CHBP}+\mathrm{NC} \operatorname{siRNA}(\mathrm{n}=3)$

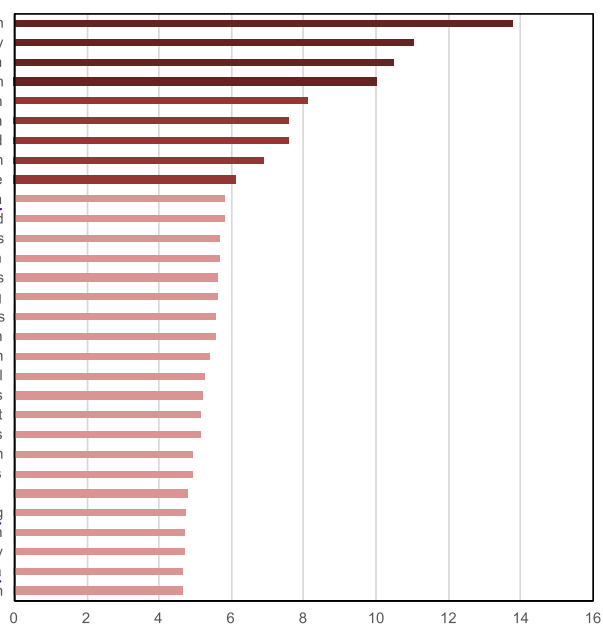

Fig. 6. GO analysis of DEGs in IR kidneys. The top 30 significantly enriched GO items of biologic processes were shown from three comparisons, modified by CHBP (A) and then further by CASP3siRNA (B) or NCsiRNA (C). The text on the left indicated the category of GO, and the bar chart indicated the enrich factor in each category. Underlined categories were particularly discussed in this study. $n=3$ animals in each group. 
reported that the proteome profile in IR kidneys at 48 hours changed by CHBP treatment was mainly related to the oxidative stress (Yang et al., 2015c). There may be differentiations between transcriptional and translational changes as well as the mouse strain (BALB/c) and dose of CHBP $(8 \mathrm{nmol} / \mathrm{kg})$.

Intriguingly, in contrast to single CHBP or CASP3siRNA treatment, cotreatment with CHBP and CASP3siRNA contributed to further preservation in renal structure, with lower active caspase-3 and HMGB1 in IR kidneys. The negative regulation of immune responses was also revealed by microarray analysis, verifying the effectiveness of further CASP3siRNA against renal IR. SLPI, among the top five DEGs upregulated by CASP3 siRNA (Table 3), was renoprotective in experimental ischemia AKI (Ochi et al., 2017). SLPI inhibits nuclear factor- $\kappa \beta$ signaling pathway (Tang et al., 2020) and the maturation of interleukin-1- $\beta$ (Zakrzewicz et al., 2019), and it was shown to be a biomarker candidate in AKI (Averdunk et al., 2019, 2020). In humans, SERPINA3, a member of the serpin superfamily of protease inhibitors, could limit inflammation by targeting cathepsin family (proinflammatory enzymes) (Horvath et al., 2005; Lannan et al., 2012). SERPINA3 expression was also found in rat kidneys, which can detect renal inflammation and fibrosis after IR injury and also serve as a urinary marker for early detection of AKI to chronic kidney disease transition (Sánchez-Navarro et al., 2019). Because murine SERPINA3M ( $F C=8.289$, Table 3 ) is a likely ortholog of human SERPINA3, the two proteins may have similar structural and kinetic characterization. The role of SERPINA3M in renal IR injury is worthy of further exploration.

Similar effects of renoprotection from CASP3siRNA were also revealed by comparing it with NCsiRNA control in CHBPtreated IR kidneys. Microarray data revealed that further CASP3siRNA treatment altered 418 DEGs or 504 DEGs in IR + CHBP kidneys without or with NCsiRNA controls, which was much higher than the 281 DEGs altered by CHBP in IR kidneys, with 46 or 9 genes in common, respectively. It was indicated that CASP3siRNA may have additive effects on renoprotection upon CHBP treatment. GO analysis identified the further altered DEGs by CASP3siRNA that were mainly linked to regulation of renal inflammation and programmed cell death upon CHBP compared with that of NCsiRNA. CFH, a negative regulator of complement alternative pathway that plays crucial roles in IR injury (Goetz et al., 2018), was increased by further CASP3siRNA treatment. Notably, ANGPTL2 was the top downregulated DEG by CASP3siRNA (Table 4). Less Angptl2 could contribute to the reduction of renal inflammation, as ANGPTL2 can activate resident macrophages and induce the secretion of proinflammatory cytokines (Umikawa et al., 2015; Amadatsu et al., 2016). Decreased ANGPTL2 may also ameliorate renal fibrosis in AKI-induced chronic kidney disease by depressing transforming growth factor- $\beta$ signaling (Morinaga et al., 2016). In addition, GREM1 was the top upregulated DEG by CASP3siRNA (Table 4), which activates vascular endothelial growth factor receptor 2 in endothelial cells to induce angiogenesis (Ravelli et al., 2013; Ji et al., 2016). The effective repair of endothelial cells in IR-injured kidney plays essential roles in maintaining the homeostasis of microvasculature and efficient renal blood flow (Kwon et al., 2010) and subsequently ameliorating tubular damage (Cantaluppi et al., 2012). The GREM1-vascular endothelial growth factor receptor 2 axis may be a novel therapeutic target for kidney inflammation and fibrosis (Mezzano et al., 2018). In addition, Yang et al., 2018 proposed that caspase-3 deficiency in mice reduced IR injury in kidneys through preserving microvascular density. However, whether the preservation of renal microvasculature in this study links to regulated GREM1 is worthy of further investigation. The above evidence indicates a promising strategy for silencing caspase- 3 and administrating CHBP at the same time for optimized outcome in improving IR injury in kidneys.

Special attention should be paid to the toxicity of NCsiRNA in the present study, which was evidenced by further elevated SCr, TID, and apoptotic levels in CHBP-modified IR kidneys. These data suggested that the synthetic siRNA duplexes may still modulate immunity and inflammation in IR kidneys, such as releasing cytokines and interferons and activating toll-like receptors on immune and nonimmune cells (Judge et al., 2005; Forsbach et al., 2008; Robbins et al., 2009). It was indicated that NCsiRNA might downregulate the influence of CHBP treatment on IR kidneys, providing an ideal and necessary control for the specific effects of CASP3siRNA in the context of CHBP-treated IR kidneys.

There are also limitations in this study. The additive renoprotective effect of CASP3siRNA upon CHBP should be further studied in the long-term prognosis of IR injury. In addition, DEGs identified by the present microarray analysis should be further validated in more comparisons with the groups, including more samples. Moreover, to select and validate potential biomarkers from identified DEGs, different downstream biologic events at the translational and posttranslational level should be further investigated in terms of dynamic expression, regulation, and intervention.

The cotreatment of CHBP and CASP3siRNA exhibited synergistic effects on preserving renal structure and reducing injury markers against 48-hour renal IR in a mouse model. The DEGs induced by CHBP are associated with the preservation of cell division, function, and metabolism, whereas the DEGs caused by CASP3siRNA are linked to improving inflammation and potential microvasculature.

\section{Acknowledgments}

We would like to acknowledge the Comparative Medicine Institute of Nantong University for providing all facilities to accommodate this study and Yaqiu Long (Key Laboratory of Receptor Research, Shanghai Institute of Materia Medica, Chinese Academy of Sciences) for providing cyclic helix B surface peptide for this study.

\section{Authorship Contributions}

Participated in research design: B. Yang.

Conducted experiments: Wu, Chen, Zhang, Liu, C. Yang, Wang. Performed data analysis: Wu.

Wrote or contributed to the writing of the manuscript: $\mathrm{Wu}, \mathrm{Zhu}$, Fan, B. Yang.

\section{References}

Amadatsu T, Morinaga J, Kawano T, Terada K, Kadomatsu T, Miyata K, Endo M, Kasamo D, Kuratsu JI, and Oike Y (2016) Macrophage-derived angiopoietin-like protein 2 exacerbates brain damage by accelerating acute inflammation after ischemia-reperfusion. PLoS One 11:e0166285.

Averdunk L, Fitzner C, Levkovich T, Leaf DE, Sobotta M, Vieten J, Ochi A, Moeckel G, Marx G, and Stoppe C (2019) Secretory leukocyte protease inhibitor (SLPI)a novel predictive biomarker of acute kidney injury after cardiac surgery: a prospective observational study. J Clin Med 8:1931.

Averdunk L, Rückbeil MV, Zarbock A, Martin L, Marx G, Jalaie H, Jacobs MJ, Stoppe C, and Gombert A (2020) SLPI - a biomarker of acute kidney injury after open and endovascular thoracoabdominal aortic aneurysm (TAAA) repair. Sci Rep 10:3453. 
Baron BW, Hyjek E, Gladstone B, Thirman MJ, and Baron JM (2010) PDCD2, a protein whose expression is repressed by BCL6, induces apoptosis in human cells by activation of the caspase cascade. Blood Cells Mol Dis 45:169-175.

Bellomo R, Kellum JA, and Ronco C (2012) Acute kidney injury. Lancet 380:756-766. Bouchard J, Acharya A, Cerda J, Maccariello ER, Madarasu RC, Tolwani AJ, Liang X, Fu P, Liu ZH, and Mehta RL (2015) A prospective international multicenter study of AKI in the intensive care unit. Clin J Am Soc Nephrol 10:1324-1331.

Brines $\mathrm{M}$ and Cerami A (2012) The receptor that tames the innate immune response. Mol Med 18:486-496.

Brines M, Patel NS, Villa P, Brines C, Mennini T, De Paola M, Erbayraktar Z, Erbayraktar S, Sepodes B, Thiemermann C, et al. (2008) Nonerythropoietic, tissueprotective peptides derived from the tertiary structure of erythropoietin. Proc Natl Acad Sci USA 105:10925-10930.

Cantaluppi V, Gatti S, Medica D, Figliolini F, Bruno S, Deregibus MC, Sordi A, Biancone L, Tetta C, and Camussi G (2012) Microvesicles derived from endothelial progenitor cells protect the kidney from ischemia-reperfusion injury by microRNAdependent reprogramming of resident renal cells. Kidney Int 82:412-427.

Chen CB, Liu LS, Zhou J, Wang XP, Han M, Jiao XY, He XS, and Yuan XP (2017) Upregulation of HMGB1 exacerbates renal ischemia-reperfusion injury by stimulating inflammatory and immune responses through the TLR4 signaling pathway in mice. Cell Physiol Biochem 41:2447-2460.

Chen SC, Guh JY, Chen HC, Yang YL, Huang JS, and Chuang LY (2007) Advanced glycation end-product-induced mitogenesis is dependent on Janus kinase 2-induced heat shock protein 70 in normal rat kidney interstitial fibroblast cells. Transl Res 149:274-281.

Dong Y, Zhang Q, Wen J, Chen T, He L, Wang Y, Yin J, Wu R, Xue R, Li S, et al. (2019) Ischemic duration and frequency determines AKI-to-CKD progression monitored by dynamic changes of tubular biomarkers in IRI mice. Front Physiol 10:153.

Forsbach A, Nemorin JG, Montino C, Müller C, Samulowitz U, Vicari AP, Jurk M, Mutwiri GK, Krieg AM, Lipford GB, et al. (2008) Identification of RNA sequence motifs stimulating sequence-specific TLR8-dependent immune responses. J Immunol 180:3729-3738.

Fragiadaki M, Lannoy M, Themanns M, Maurer B, Leonhard WN, Peters DJ, Moriggl R, and Ong AC (2017) STAT5 drives abnormal proliferation in autosomal dominant polycystic kidney disease. Kidney Int 91:575-586.

Gobe GC, Bennett NC, West M, Colditz P, Brown L, Vesey DA, and Johnson DW (2014) Increased progression to kidney fibrosis after erythropoietin is used as a treatment for acute kidney injury. Am J Physiol Renal Physiol 306:F681-F692.

Goetz L, Laskowski J, Renner B, Pickering MC, Kulik L, Klawitter J, Stites E, Christians U, van der Vlag J, Ravichandran K, et al. (2018) Complement factor H protects mice from ischemic acute kidney injury but is not critical for controlling complement activation by glomerular IgM. Eur J Immunol 48:791-802.

Harris MA, Clark J, Ireland A, Lomax J, Ashburner M, Foulger R, Eilbeck K, Lewis S, Marshall B, Mungall C, et al.; Gene Ontology Consortium (2004) The Gene Ontology (GO) database and informatics resource. Nucleic Acids Res 32:D258-D261.

Hawgood S, Hook-Barnard IG, O'Brien TC, and Yamamoto KR (2015) Precision medicine: beyond the inflection point. Sci Transl Med 7:300ps17.

Horvath AJ, Irving JA, Rossjohn J, Law RH, Bottomley SP, Quinsey NS, Pike RN, Coughlin PB, and Whisstock JC (2005) The murine orthologue of human antichymotrypsin: a structural paradigm for clade A3 serpins. J Biol Chem 280:43168-43178.

Ji C, Huang JW, Xu QY, Zhang J, Lin MT, Tu Y, He L, Bi ZG, and Cheng B (2016) Gremlin inhibits UV-induced skin cell damages via activating VEGFR2-Nrf2 signaling. Oncotarget 7:84748-84757.

Judge AD, Sood V, Shaw JR, Fang D, McClintock K, and MacLachlan I (2005) Sequence-dependent stimulation of the mammalian innate immune response by synthetic siRNA. Nat Biotechnol 23:457-462.

Kaushal GP and Shah SV (2016) Autophagy in acute kidney injury. Kidney Int 89: 779-791.

Kwon O, Miller S, Li N, Khan A, Kadry Z, and Uemura T (2010) Bone marrowderived endothelial progenitor cells and endothelial cells may contribute to endothelial repair in the kidney immediately after ischemia-reperfusion. $J$ Histochem Cytochem 58:687-694.

Lannan EA, Galliher-Beckley AJ, Scoltock AB, and Cidlowski JA (2012) Proinflammatory actions of glucocorticoids: glucocorticoids and TNF $\alpha$ coregulate gene expression in vitro and in vivo. Endocrinology 153:3701-3712.

Li Y, Hou D, Chen X, Zhu J, Zhang R, Sun W, Li P, Tian Y, and Kong X (2019) Hydralazine protects against renal ischemia-reperfusion injury in rats. Eur $J$ Pharmacol 843:199-209.

Mehta RL, Burdmann EA, Cerdá J, Feehally J, Finkelstein F, García-García G, Godin M, Jha V, Lameire NH, Levin NW, et al. (2016) Recognition and management of acute kidney injury in the International Society of Nephrology 0by25 Global Snapshot: a multinational cross-sectional study. Lancet 387:2017-2025.

Mehta RL, Cerdá J, Burdmann EA, Tonelli M, García-García G, Jha V, Susantitaphong P, Rocco M, Vanholder R, Sever MS, et al. (2015) International Society of Nephrology's 0by25 initiative for acute kidney injury (zero preventable deaths by 2025): a human rights case for nephrology. Lancet 385:2616-2643.

Mezzano S, Droguett A, Lavoz C, Krall P, Egido J, and Ruiz-Ortega M (2018) Gremlin and renal diseases: ready to jump the fence to clinical utility? Nephrol Dial Transplant 33:735-741.

Moore PK, Hsu RK, and Liu KD (2018) Management of acute kidney injury: core curriculum 2018. Am J Kidney Dis 72:136-148.

Morinaga J, Kadomatsu T, Miyata K, Endo M, Terada K, Tian Z, Sugizaki T, Tanigawa $\mathrm{H}$, Zhao J, Zhu S, et al. (2016) Angiopoietin-like protein 2 increases renal fibrosis by accelerating transforming growth factor- $\beta$ signaling in chronic kidney disease. Kidney Int 89:327-341.

Noble RA, Lucas BJ, and Selby NM (2020) Long-term outcomes in patients with acute kidney injury. Clin J Am Soc Nephrol 15:423-429.

Ochi A, Chen D, Schulte W, Leng L, Moeckel N, Piecychna M, Averdunk L, Stoppe C Bucala R, and Moeckel G (2017) MIF-2/D-DT enhances proximal tubular cell regeneration through SLPI- and ATF4-dependent mechanisms. Am J Physiol Renal Physiol 313:F767-F780.
Patel NS, Kerr-Peterson HL, Brines M, Collino M, Rogazzo M, Fantozzi R, Wood EG, Johnson FL, Yaqoob MM, Cerami A, et al. (2012) Delayed administration of pyroglutamate helix B surface peptide (pHBSP), a novel nonerythropoietic analog of erythropoietin, attenuates acute kidney injury. Mol Med 18:719-727.

Ravelli C, Mitola S, Corsini M, and Presta M (2013) Involvement of $\alpha \mathrm{v} \beta 3$ integrin in gremlin-induced angiogenesis. Angiogenesis 16:235-243.

Robbins M, Judge A, and MacLachlan I (2009) siRNA and innate immunity. Oligonucleotides 19:89-102.

Sánchez-Navarro A, Mejía-Vilet JM, Pérez-Villalva R, Carrillo-Pérez DL, MarquinaCastillo B, Gamba G, and Bobadilla NA (2019) SerpinA3 in the early recognition of acute kidney injury to chronic kidney disease (CKD) transition in the rat and its potentiality in the recognition of patients with CKD. Sci Rep 9:10350.

Shen H, Liu T, Morse BL, Zhao Y, Zhang Y, Qiu X, Chen C, Lewin AC, Wang XT, Liu G, et al. (2015) Characterization of organic anion transporter 2 (SLC22A7): a highly efficient transporter for creatinine and species-dependent renal tubular expression. Drug Metab Dispos 43:984-993.

Shi M, Flores B, Li P, Gillings N, McMillan KL, Ye J, Huang LJ, Sidhu SS, Zhong YP, Grompe MT, et al. (2018) Effects of erythropoietin receptor activity on angiogenesis, tubular injury, and fibrosis in acute kidney injury: a "U-shaped" relationship. Am J Physiol Renal Physiol 314:F501-F516.

Tang R, Botchway BOA, Meng Y, Zhang Y, Zhou C, Jiang J, and Liu X (2020) The inhibition of inflammatory signaling pathway by secretory leukocyte protease inhibitor can improve spinal cord injury. Cell Mol Neurobiol DOI: 10.1007/s10571020-00799-1 [published ahead of print].

Umikawa M, Umikawa A, Asato T, Takei K, Matsuzaki G, Kariya K, and Zhang CC (2015) Angiopoietin-like protein 2 induces proinflammatory responses in peritoneal cells. Biochem Biophys Res Commun 467:235-241.

Wang S, Zhang C, Li J, Niyazi S, Zheng L, Xu M, Rong R, Yang C, and Zhu T (2017) Erythropoietin protects against rhabdomyolysis-induced acute kidney injury by modulating macrophage polarization. Cell Death Dis 8:e2725.

Wegner W, Burckhardt G, and Henjakovic M (2014) Transcriptional regulation of human organic anion transporter 1 by B-cell CLL/lymphoma 6. Am J Physiol Renal Physiol 307:F1283-F1291.

Wei Q, Xiao X, Fogle P, and Dong Z (2014) Changes in metabolic profiles during acute kidney injury and recovery following ischemia/reperfusion. PLoS One 9:e106647.

Wu H, Ma J, Wang P, Corpuz TM, Panchapakesan U, Wyburn KR, and Chadban SJ (2010) HMGB1 contributes to kidney ischemia reperfusion injury. J Am Soc Nephrol 21:1878-1890.

Wu Y, Zhang J, Liu F, Yang C, Zhang Y, Liu A, Shi L, Wu Y, Zhu T, Nicholson ML, et al. (2013) Protective effects of HBSP on ischemia reperfusion and cyclosporine a induced renal injury. Clin Dev Immunol 2013:758159.

Yang B, Hosgood SA, Bagul A, Waller HL, and Nicholson ML (2011a) Erythropoietin regulates apoptosis, inflammation and tissue remodelling via caspase- 3 and IL-1 $\beta$ in isolated hemoperfused kidneys. Eur J Pharmacol 660:420-430.

Yang B, Hosgood SA, and Nicholson ML (2011b) Naked small interfering RNA of caspase-3 in preservation solution and autologous blood perfusate protects isolated ischemic porcine kidneys. Transplantation 91:501-507.

Yang B, Lan S, Dieudé M, Sabo-Vatasescu JP, Karakeussian-Rimbaud A, Turgeon J, Qi S, Gunaratnam L, Patey N, and Hébert MJ (2018) Caspase-3 is a pivotal regulator of microvascular rarefaction and renal fibrosis after ischemia-reperfusion injury. J Am Soc Nephrol 29:1900-1916.

Yang C, Cao Y, Zhang Y, Li L, Xu M, Long Y, Rong R, and Zhu T (2015a) Cyclic helix $\mathrm{B}$ peptide inhibits ischemia reperfusion-induced renal fibrosis via the PI3K/Akt/ FoxO3a pathway. J Transl Med 13:355.

Yang C, Hosgood SA, Meeta P, Long Y, Zhu T, Nicholson ML, and Yang B (2015b) Cyclic helix $\mathrm{B}$ peptide in preservation solution and autologous blood perfusate ameliorates ischemia-reperfusion injury in isolated porcine kidneys. Transplant Direct 1:e6.

Yang C, Liu J, Li L, Hu M, Long Y, Liu X, Zhu T, Huang X, Zhao S, Liu S, et al. (2015c) Proteome analysis of renoprotection mediated by a novel cyclic helix B peptide in acute kidney injury. Sci Rep 5:18045.

Yang C, Xu Z, Zhao Z, Li L, Zhao T, Peng D, Xu M, Rong R, Long YQ, and Zhu T (2014a) A novel proteolysis-resistant cyclic helix B peptide ameliorates kidney ischemia reperfusion injury. Biochim Biophys Acta 1842:2306-2317.

Yang C, Zhao T, Lin M, Zhao Z, Hu L, Jia Y, Xue Y, Xu M, Tang Q, Yang B, et al. (2013) Helix B surface peptide administered after insult of ischemia reperfusion improved renal function, structure and apoptosis through beta common receptor/ erythropoietin receptor and PI3K/Akt pathway in a murine model. Exp Biol Med (Maywood) 238:111-119

Yang C, Zhao T, Zhao Z, Jia Y, Li L, Zhang Y, Song M, Rong R, Xu M, Nicholson ML, et al. (2014b) Serum-stabilized naked caspase-3 siRNA protects autotransplant kidneys in a porcine model. Mol Ther 22:1817-1828.

Yang L, Xing G, Wang L, Wu Y, Li S, Xu G, He Q, Chen J, Chen M, Liu X, et al.; ISN AKF 0by25 China Consortiums (2015d) Acute kidney injury in China: a crosssectional survey, Lancet 386:1465-1471.

Zakrzewicz A, Richter K, Zakrzewicz D, Siebers K, Damm J, Agné A, Hecker A McIntosh JM, Chamulitrat W, Krasteva-Christ G, et al. (2019) SLPI inhibits ATPmediated maturation of IL-1 $\beta$ in human monocytic leukocytes: a novel function of an old player. Front Immunol 10:664.

Zeng Y, Zheng L, Yang Z, Yang C, Zhang Y, Li J, Zhang W, Zhang M, Hu M, Wang S, et al. (2017) Protective effects of cyclic helix B peptide on aristolochic acid induced acute kidney injury. Biomed Pharmacother 94:1167-1175.

Zhang Y, Wang Q, Liu A, Wu Y, Liu F, Wang H, Zhu T, Fan Y, and Yang B (2020) Erythropoietin derived peptide improved endoplasmic reticulum stress and ischemia-reperfusion related cellular and renal injury. Front Med (Lausanne) 7:5.

Address correspondence to: Bin Yang, Renal Group, Cardiovascular Sciences, University of Leicester, University Hospitals of Leicester, University Rd., Leicester LE1 7RH, UK. E-mail: by5@le.ac.uk or dryangbin@hotmail.com 